\title{
Educação em Saúde em Grupo no Tratamento de Obesos Grau III: um Desafio para os Profissionais de Saúde
}

\section{Group Health Education in the Treatment of Obese Class III: a Challenge for Health Professionals}

Raquel Lima Soeiro ${ }^{\circledR \odot}$

Geilsa Soraia Cavalcanti Valente

Elaine Antunes Cortez ${ }^{1 \odot}$

Livia Mendes Mesquita ${ }^{I \oplus}$

Simone Costa da Matta Xavier ${ }^{1 \odot}$

Bianca Maria Innocencio da Silveira Lobo ${ }^{\text {ॠ }}$

\section{PALAVRAS-CHAVE}

- Obesidade mórbida.

- Enfermagem.

- Educação em saúde.

- Atenção primária a saúde.
A obesidade tem se apresentado como sério problema de saúde pública mundial devido à magnitude que seus números alcançaram nos últimos anos. Diante do quadro de aumento do sobrepeso e obesidade no município do Rio de Janeiro, a Secretaria Municipal de Saúde do Rio de Janeiro (SMSRJ), com o apoio técnico do Instituto de Nutrição Annes Dias (INAD), implementou em julho de 2011 o primeiro Centro de Referência em Obesidade (CRO) no bairro de Acari. Este serviço oferece tratamento clínico ambulatorial para usuários com obesidade grau III (também conhecida como obesidade mórbida), com consultas individuais, assim como atendimentos em grupo, e também funciona como apoio especializado, contribuindo para ampliação da capacidade de atuação das equipes da Atenção Primária à Saúde (APS) com esse perfil de usuários através de uma equipe multiprofissional composta por educador físico, enfermeiro, médico endocrinologista, nutricionista e psicólogo. Neste contexto, a partir das experiências vivenciadas no CRO o objetivo do estudo é realizar uma revisão da literatura sobre a importância das atividades de educação em saúde em grupo no tratamento de indivíduos obesos grau III e como os profissionais de saúde tem lidado com esse tema nas publicações dos últimos 5 anos. Metodologia: Trata-se de uma revisão integrativa da literatura, de acordo com a estratégia PICO (participantes, intervenção, contexto e resultados), cuja coleta dos dados foi realizada entre outubro e novembro de 2018, nas bases de dados Literatura Latino Americana e do Caribe em Ciências da Saúde (LILACS), Web of science, SciVerse Scopus, PUBMED e Bases de Dados em Enfermagem (BDENF). Resultados: A partir da análise dos 11 artigos selecionados no estudo foi possivel identificar três temáticas relevantes para a discussão: Rede de apoio aos usuários em tratamento, A Atenção primária à saúde e atividades em grupo no tratamento de obesos grau III, implementação de serviços especializados e qualificados no atendimento desse público. Conclusão: As evidências mostram que a função da equipe de saúde em relação ao cuidado desse perfil de usuários vai além de orientações e informações e que o desenvolvimento e a implementação de serviços e/ou tratamentos voltados para obesos grau III constituem desafios significativos, e necessitam de profissionais qualificados na configuração de equipe multidisciplinar atuando de forma interdisciplinar que conscientizem o usuário a assumir o protagonismo de seu tratamento. E para isso, as atividades educativas em saúde em grupo, são ferramentas importantes para promoção de autonomia e compartilhamentos de experiências na busca por qualidade de vida relacionada à saúde. 


\section{KEY-WORDS}

- Morbid obesity.

- Nursing

- Education in Health

- Primary Health Care

\begin{abstract}
Obesity has become a serious, global public health problem, with the number of people it affects reaching extreme numbers in recent years.In view of the increase in overweight and obese individuals in the municipality of Rio de Janeiro, the Municipal Health Department of Rio de Janeiro (SMSRJ), with the technical support of the Annes Dias Institute of Nutrition (INAD), implemented the first Obesity Reference Centre (CRO) in the district of Acari in July 2011.This service offers outpatient clinical treatment for class III obesity users (also known as morbid obesity), with individual consultations, as well as group visits. It also represents a specialized support service, contributing toward the increased capacity of Primary Health Care (PHC) teams to offer assistance to users with this profile through a cross-functional team composed of a physical education instructor, nurse, endocrinologist, nutritionist and psychologist.In this context, based on the experiences of the CRO, the objective of the study is to perform a literature review on the importance of group health education activities in the treatment of class III obese individuals and how health professionals have addressed this topic in publications of the last five years.Methodology:This is an integrative literature review, according to the PICO strategy (participants, intervention, context and results), the data of which was collected between October and November 2018, in the Latin American Literature and Caribbean in Health Sciences (Lilacs), Web of Science, SciVerse Scopus, Pubmed databases and Nursing Databases (BDENF). Results:Based on the analysis of the 11 articles selected in the study, three relevant themes were identified for discussion:network of users in treatment, primary health care and group activities in the treatment of class III obesity, implementation of specialized and qualified service to attend this group of users.Conclusion:The evidence shows that the role of the health team in relation to the care of this user profile goes beyond guidelines and information and that the development and implementation of services and/or treatments for class III obesity are significant challenges and require qualified professionals to compose multidisciplinary teams acting in an interdisciplinary manner that make the user aware that he or she must take the lead role in their own treatment. And to this end, group health education activities are important tools to promote autonomy and share experiences in the search for health-related quality of life.
\end{abstract}

Recebido em 9/4/19

Aceito em 20/5/19

\section{INTRODUÇÃO}

Devido à magnitude que os números da obesidade têm alcançado nos últimos anos, essa doença crônica constitui um sério problema de saúde pública no mundo,o que representa um importante fator de risco para o surgimento de outras Doenças Crônicas Não Transmissíveis (DCNTs). Atualmente, mais de 1,9 bilhão de adultos apresentam excesso de peso. Destes, mais de 650 milhões são obesos, o que representa 39\% da população mundial ${ }^{1}$.

No Brasil, o levantamento de 2018 da Vigilância de Fatores de Risco e Proteção para Doenças Crônicas por Inquérito Telefônico (Vigitel)- pesquisa telefônica realizada com maiores de 18 anos nas 26 capitais e no Distrito Federal sobre diversos assuntos relacionados à saúde - aponta que $19,8 \%$ da população brasileira são obesos, sendo que em 2010 esse percentual era de $15 \%$. A pesquisa mostra também que mais da metade da população adulta brasileira $(55,7 \%)$ está com excesso de peso. ${ }^{2}$

O Rio de Janeiro, entre as capitais brasileiras e Distrito Federal, ocupa o sétimo lugar no ranking das cidades com maior número de indivíduos com excesso de peso $(57,7 \%)$ e o segundo lugar na concentração de indivíduos obesos $(22,4 \%)^{2}$.

Para reverter esse crescimento da prevalência da obesidade, os serviços de saúde são estimulados a implementar e inovar estratégias para prevenção e enfrentamento da obesidade e promoção da saúde, assim como a organizar novos serviços especializados que contribuam para o controle e reabilitação desses casos na Atenção Primária à Saúde (APS) 3 .

A APS é um local privilegiado para a promoção de saúde por ser a principal porta de entrada dos usuários no sistema de saúde e, no caso da obesidade, para o enfrentamento dessa 
doença, além do cuidado das consequências que esse excesso de peso causa nesses indivíduos. As equipes multiprofissionais são de suma importância nesse cenário, e o enfermeiro na atenção primária à saúde tem uma atuação fundamental nesse nível de atenção,por estabelecer a estratificação do risco para obesidade, incentivar o autocuidado e o protagonismo do usuário em seu tratamento, garantir o apoio necessário aos usuários do Sistema Único de Saúde (SUS) com obesidade, assim como àqueles em risco de desenvolvê-la, realizar ações de promoção à saúde com participação social, assim como promover a prestação de cuidado aos indivíduos que já se encontram com obesidade, principalmente se houver comorbidades associadas, e acompanhar aqueles que se submeteram a procedimentos cirúrgicos relacionados à obesidade ${ }^{4}$.

Neste contexto de cuidado, é necessário visar ao enfrentamento das dificuldades no manejo dessa doença crônica, ou seja, as ações de promoção de saúde, prevenção e controle de agravos resultantes da obesidade devem ser rotineiras ${ }^{5}$.

Em razão do aumento significativo da ocorrência de sobrepeso e obesidade na cidade do Rio de Janeiro, a Secretaria Municipal de Saúde do Rio de Janeiro (SMSRJ), contando com o apoio técnico do Instituto de Nutrição Annes Dias (Inad), implementou o primeiro Centro de Referência em Obesidade (CRO) no bairro de Acari em julho de 2011. O serviço, articulado com a Estratégia de Saúde da Família, oferece tratamento clínico ambulatorial a usuários com obesidade grau III (também nomeada como obesidade mórbida) por meio de uma equipe multiprofissional composta por psicólogo, educador físico, enfermeiro, médico endocrinologista e nutricionista.

A Organização Mundial da Saúde (OMS), cujas preconizações também são adotadas pelo Conselho Latino-Americano de Obesidade e Ministério da Saúde, classifica a obesidade em três níveis de gravidade com base no $\mathrm{IMC}^{6}$ : obesidade grau I, quando o IMC se situa entre 30 e $34,9 \mathrm{~kg} / \mathrm{m}^{2}$; obesidade grau II, com IMC entre 35 e $39,9 \mathrm{~kg} / \mathrm{m}^{2}$; e obesidade grau III ou obesidade mórbida, quando o IMC ultrapassa $40 \mathrm{~kg} / \mathrm{m}^{2}$.

O Índice de Massa Corporal (IMC), também conhecido como Índice de Quelet, é o critério mais utilizado no mundo para a avaliação nutricional por ser uma técnica não invasiva e de fácil realização. O IMC é obtido dividindo-se o peso do corpo em quilogramas pela altura em metros quadrados $\left(\mathrm{kg} / \mathrm{m}^{2}\right)^{7}$.

Os critérios para a entrada no CRO foram definidos com base nessa classificação dos níveis de obesidade, tendo como ponto de corte para a admissão no tratamento pessoas obesas que apresentam IMC superior a $40 \mathrm{~kg} / \mathrm{m}^{2}$ associado ao diagnóstico de diabetes mellitus ou IMC superior a $50 \mathrm{~kg} / \mathrm{m}^{2}$ independentemente de existirem comorbidades associadas.Esses critérios têm por objetivo oferecer assistência a um segmento mais grave da obesidade.

Atualmente, o fluxo de entrada e acompanhamento dos usuários está representado na Figura 1. A porta de entrada do serviço é o "Grupo de Acolhimento", que tem o enfermeiro como principal referência para a organização e condução do grupo, onde este recebe a retaguarda de outros profissionais quanto a avaliação antropométrica, abertura de prontuário e eventuais contrarreferências daqueles que estiverem fora dos critérios de inclusão no serviço. Neste grupo é observado se os usuários encaminhados atendem aos critérios de inclusão e são esclarecidos os objetivos, funcionamento e principais dúvidas sobre o serviço e o acompanhamento clínico. Os usuários inseridos no programa participam de encontros no chamado "Grupo de Conhecimento em Saúde", cujo objetivo é orientar sobre a obesidade e as principais comorbidades, tratamentos medicamentosos e a importância de mudança de estilo de vida. Todos passam por avaliação com todos os profissionais e, em paralelo às consultas individuais, os usuários participam dos grupos multiprofissionais e oficinas, como o "Grupo Saúde em Foco", que aborda questões gerais sobre saúde, o "Grupo Corpo em Movimento", voltado à atividade física, o "Grupo Terapêutico", coordenado pelo psicólogo, e as "Oficinas Culinárias".

As atividades em grupo são uma das estratégias para o tratamento da obesidade adotadas no CRO consideradas de grande importância, em virtude de sua potência em promover troca de vivências, dificuldades enfrentadas, experiências adquiridas e construção de estratégias que diminuam o isolamento social. Estas também aliam a menor utilização de recursos, o alcance de maior número de indivíduos e a criação de um ambiente de apoio num espaço em que os usuários dividem objetivos e experiências.

O grupo não corresponde apenas a um somatório de indivíduos, mas compreende um conjunto de pessoas interdependentes, movidas por necessidades semelhantes que se reúnem na tentativa de alcançar objetivos comuns ${ }^{8}$. No que diz respeito aos aspectos sociais, subjetivos e biológicos, os grupos são vistos como um espaço no qual é possível trabalhar essas diferentes faces do ser humano. Desta forma, as atividades em grupo são fundamentais tanto para o indivíduo quanto para a comunidade em que este se encontra inserido?

Cabe ressaltar que, além de atender a essas demandas, os CROs estão disponíveis para discussão de casos e matriciamento de equipes da ESF, entre outras atividades. O Apoio Matricial, também chamado de matriciamento, tem sido uma metodologia de trabalho preconizada na APS, na medida em que se propõe a assegurar retaguarda especializada assistencial e suporte técnico-pedagógico às equipes de referência ${ }^{10}$. 
Figura 1

Fluxo de entrada e acompanhamento dos usuários no CRO

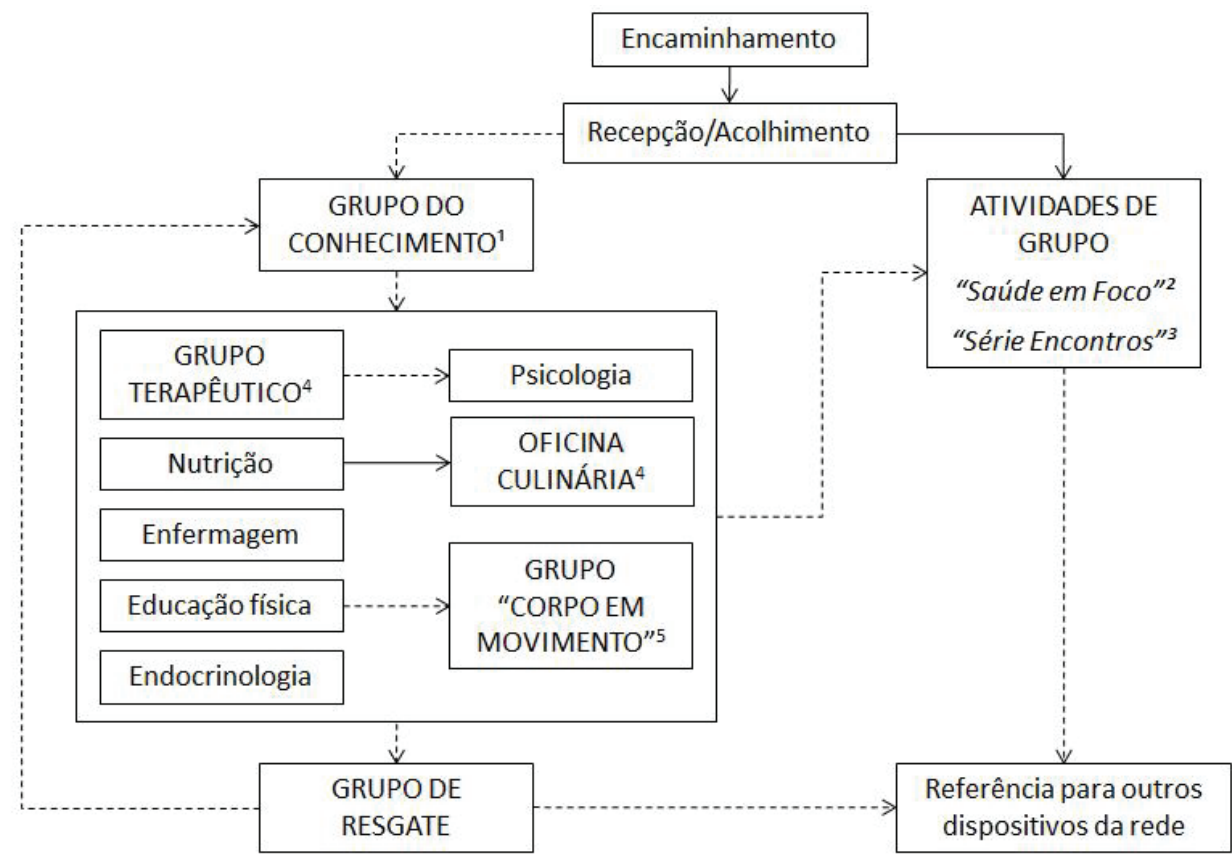

${ }^{1}$ Grupo mensal aberto aos pacientes com critérios de admissão no CRO;

Temas fixos e rotativos: Hipertensão e diabetes; Alimentação saudável; Atividade física; Ansiedade; Autocuidado

${ }^{2}$ Grupo quinzenal aberto a todos os interessados; Temas diversos

${ }^{3}$ Grupo com frequência definida em calendário semestral, aberto a todos os interessados

${ }^{4}$ Grupos quinzenais abertos aos pacientes com critérios de admissão no CRO

${ }^{5} \mathrm{Grupo}$ semanal aberto aos pacientes admitidos no CRO e considerados aptos para atividade física

Na prevenção e no tratamento do sobrepeso/obesidade, o apoio matricial interdisciplinar é um grande potencializador da resolutividade das equipes da APS, pois promove a ampliação dos saberes acerca da complexidade desses agravos e permite melhor oferta de cuidados ${ }^{11}$.

Levando-se em consideração que a o CRO é composto de equipe multiprofissional, ele funciona como um apoio especializado, contribuindo para ampliar a capacidade de atuação das equipes da saúde da família com os usuários obesos grau III.

Assim, profissionais da rede de APS do Rio de Janeiro buscam o CRO para auxiliar na criação e implementação de grupos com obesos de suas áreas. Por vezes, os profissionais do CRO foram convidados a participar de reuniões para discutir o manejo desses usuários e a construção de projetos terapêuticos. Nesse contexto, as atividades em grupo têm forte relevância e despertam nos profissionais algumas questões, como, por exemplo: como motivá-los a se deslocarem de casa para o grupo, quais temas discutir nesses encontros, como orientar atividade física e como mantê-los ativos e presentes nesses grupos devido às dificuldades que a obesidade impõe. Torna-se importante, dessa forma, a capacitação dessas equipes para desenvolverem estratégias de enfrentamento dessas questões em seus territórios.

Nesse contexto ecom base nas experiências vivenciadas no $\mathrm{CRO}$, o presente estudo teve como objetivo fazer uma revisão da literatura sobre a importância das atividades de educação em saúde em grupo no tratamento de indivíduos obesos grau III e como os profissionais de saúde têm lidado com esse tema.

\section{MÉTODO}

Trata-se de uma revisão integrativa (RI) para reunir e sintetizar achados de estudos realizados, com o intuito de aprofundar o conhecimento sobre a importância das atividades de educação em saúde em grupo no tratamento de indivíduos obesos grau III e verificar como os profissionais de saúde têm lidado com esse tema. Para buscar a relevância deste estudo, 
realizou-se uma busca bibliográfica para aferir os artigos sobre o tema proposto com o intuito de contribuir para aprofundar o conhecimento relativo ao assunto. A pesquisa foi conduzida em duas fases: (a) triagem de títulos e resumos: nesta fase, foram excluídos os artigos que não se adequavam à temática estudada; (b) após a primeira triagem dos títulos e resumos, foi verificada a existência de duplicidade dos artigos nas seleções das bases de dados, ou seja, se dois artigos iguais foram selecionados em bases de dados diferentes. Após essas duas triagens, os artigos selecionados foram lidos integralmente para a construção deste trabalho.

Com a finalidade de atribuir rigor metodológico, esta revisão foi realizada em seis fases: elaboração da pergunta norteadora; busca ou amostragem na literatura; coleta de dados (definição dos sujeitos, metodologia, tamanho da amostra, mensuração de variáveis, método de análise e conceitos embasadores empregados); análise crítica dos estudos incluídos; discussão dos resultados; apresentação da revisão integrati$\mathrm{va}^{12}$. Para guiar a presente revisão integrativa formulou-se a seguinte questão: quais as evidências científicas na literatura sobre a importância das atividades em grupo no tratamento de obesos grau III na atenção primária à saúde?

$\mathrm{Na}$ estruturação desta questão, recorremos à estratégia $\mathrm{Pico}^{13}$ : participantes; intervenção; contexto do estudo e resultados (outcomes).

A coleta de dados ocorreu de outubro a novembro de 2018 nas bases de dados Literatura Latino-Americana e do Caribe em Ciências da Saúde (Lilacs),WebofScience, SciVerse Scopus, Pubmed e Bases de Dados em Enfermagem (BDENF).

Foram utilizados os seguintes descritores para busca dos artigos e suas combinações nas línguas portuguesa e inglesa: Obesidade mórbida, Enfermagem, Educação em saúde, Edu- cação continuada e Atenção primária e os MeSH: Morbid Obesity, Nursing, Health Education,Continuing Education e Primary Health Care. Os operadores booleanos "OR" e "AND" foram utilizados com a intenção de aproximar os objetivos da pesquisa do levantamento bibliográfico encontrado.

Os critérios de inclusão definidos para a seleção dos artigos foram: artigos publicados em português, inglês e espanhol; artigos na íntegra que retratassem a temática referente a tratamento de obesos grau III adultos por meio de grupos de educação em saúde e artigos publicados e indexados nos referidos bancos de dados nos últimos cinco anos.

\section{RESULTADOS}

Somando-se todas as bases de dados, foram encontrados 1.269 artigos. A Tabela 1 indica o caminho metodológico percorrido para a escolha dos artigos por meio dos descritores associados e o quantitativo de produções bibliográficas a ser analisado.

Assim, após a seleção das produções bibliográficas consideradas relevantes, aplicação dos critérios de inclusão e exclusão de estudos evidenciados em duplicidade nas diferentes bases de dados, obtivemos 11 artigos como amostra potencial para análise. As informações extraídas de cada artigo foram categorizadas no Quadro1, que apresenta as características e as principais conclusões de cada estudo.

Após leitura e análise minuciosa dos artigos selecionados, foi possível extrair deles as evidências relativas acomo acontece o tratamento para obesos grau III na atenção primária e a importância das atividades de educação em saúde em grupo no cuidado desses usuários. Nesta fase, para melhor organização da análise, por meio de uma leitura exploratória de cada artigo, foram identificadas, traduzidas e transcritas frases e palavras que correspondiam a elementos de interesse relacio-

\begin{tabular}{|c|c|c|c|c|c|}
\hline \multicolumn{6}{|c|}{$\begin{array}{c}\text { TABELA } 1 \\
\text { Estratégias de busca, banco de dados e publicações encontradas - ano de } 2018\end{array}$} \\
\hline DESCRITORES & $\begin{array}{l}\text { BASE DE } \\
\text { DADOS }\end{array}$ & $\begin{array}{l}\text { PUBLICAÇÕES } \\
\text { ENCONTRADAS }\end{array}$ & $\begin{array}{l}\text { ARTIGOS } \\
\text { SELECIONADOS } \\
\text { CRITÉRIOS DE } \\
\text { INCLUSÃO }\end{array}$ & $\begin{array}{l}\text { ARTIGOS } \\
\text { EXLUCÍDOS } \\
\text { APÓS LEITURA } \\
\text { DE TÍTULOS, } \\
\text { RESUMOS E } \\
\text { REPETIDOS }\end{array}$ & $\begin{array}{l}\text { SELECIONADOS DE } \\
\text { ACORDO COM O } \\
\text { OBJETO DO ESTUDO }\end{array}$ \\
\hline \multirow{3}{*}{$\begin{array}{c}\text { Obesidade mórbida OR } \\
\text { Obesidade grau III AND } \\
\text { Enfermagem AND Educação } \\
\text { continuada AND Educação em } \\
\text { saúde AND Atenção primária }\end{array}$} & $\begin{array}{l}\text { MEDLINE/ } \\
\text { PUBMED }\end{array}$ & 427 & 100 & 92 & 8 \\
\hline & LILACS & 30 & 18 & 18 & 0 \\
\hline & BDENF & 315 & 31 & 30 & 1 \\
\hline $\begin{array}{l}\text { Morbid Obesity AND Nursing } \\
\text { AND Continuing education }\end{array}$ & SCOPUS & 370 & 57 & 57 & 0 \\
\hline $\begin{array}{l}\text { AND Health Education AND } \\
\text { Primary Health Care }\end{array}$ & Web Of Science & 127 & 22 & 20 & 2 \\
\hline
\end{tabular}

Fonte: Elaboração própria. 


\begin{tabular}{|c|c|c|c|c|}
\hline \multicolumn{5}{|c|}{$\begin{array}{l}\text { QUADRO } 1 \\
\text { gos da Revisão Integrativa }\end{array}$} \\
\hline Título & Autores & Base de dados/fonte & Ano & Conclusões \\
\hline $\begin{array}{l}\text { Predictors of physical } \\
\text { and mental health in } \\
\text { persons with morbid } \\
\text { obesity attending a patient } \\
\text { education course - a two- } \\
\text { year follow-up study }\end{array}$ & Lerdalet al. ${ }^{14}$ & $\begin{array}{l}\text { Pubmed } \\
\text { Health Qual Life } \\
\text { Outcomes }\end{array}$ & 2017 & $\begin{array}{l}\text { Pessoas com obesidade mórbida à espera de cirurgia bariátrica tiveram a saúde } \\
\text { melhorada após terem frequentado um curso educacional durante dois anos cujo } \\
\text { objetivo era ajudar os participantes a tomar decisões mais informadas sobre a } \\
\text { opção de cirurgia ou tratamento alternativopara alcançar um estilo de vida mais } \\
\text { saudável e, em seguida, melhorar sua qualidade de vida. }\end{array}$ \\
\hline
\end{tabular}

The impact of

severe obesity on healthcare Espallardo et al..$^{15}$

Pubmed

ObesitySurgery

O estudo fornece evidências do fato de que a obesidade grave, a obesidade e a morbidade estão tendo um impacto significativo no uso de recursos de saúde na resource utilisation in

The support needs of patients waiting for

Pubmed quando comparadas com outros grupos de IMC. Os resultados sugerem a importância de ações que visem prevenir e tratar a obesidade, especialmente os casos mais graves.

A esperapela cirurgia bariátrica parece estar comumente associada ao ganho de peso e ao declínio fisiológico ou psicológico da saúde. Remediar os déficits de comunicação efortalecer a rede de apoio pode melhorar a experiência ou os resultados da espera pelos pacientes e conferir ganhos de qualidade de vida, independentemente da perda de peso. publicly funded bariatric surgery - implications for Sharman et al ${ }^{16}$ health service planners

Expectations and patients' experiences of obesity prior to bariatric surgery: a qualitative study

$\begin{array}{lc}\text { Homeret al. } & \text { Pubmed } \\ & \text { BMJ open }\end{array}$

The impact of a lifestyle change program on the mental health of obese under-served African American women

The value of health and weight loss among primary care patients with moderate to severe obesity: do quality of life factors have a larger influence than comorbidities?

Evaluation of a multidisciplinary Tier 3 weight management service for adults with morbid obesity, or obesity and comorbidities, based in primary care

\section{Access to weight} reduction interventions for overweight and obese patients in UK primary care: population-based cohort study

Trajectories of physical and mental health among persons with morbid obesity and persons with COPD: a longitudinal comparative study
Pubmed

Groh e Urbancic ${ }^{18} \quad$ Archives of Psychiatric 2015 Nursing

O estudo destaca a importância do controle de peso e serviços que avaliam e modificam as expectativas do paciente. A importância de fornecermudança de comportamento e apoio à mudança e ao autogerenciamento também é enfatizada, e este apoio deve levar em conta o impacto da estigmatização e vergonha quesão maximizadas após a cirurgia.

O estudo contribui para a literatura atual por mostrar a relação entre um programa de mudança de estilo de vida e resultados de saúde mental, pois mesmo uma modesta perda de peso pode facilitar a melhoria desta. Além disso, demonstra que mulheres afro-americanas, uma população sub-representada no estilo de vida,estão tão empenhadas em melhorar a sua saúde quanto mulheres afluentes, apesar da pobreza e das barreiras que encontram no dia a dia.

Como amanutenção da perda de peso é difícil e requer compromisso do paciente, é importante trabalhar com os pacientes para que acreditem que vale a pena manter a perda de peso que trabalharam tão arduamente para conseguir. Os pa-

Wee et al. ${ }^{19} \quad$ Pubmed

Obes Res Clin Pract.

2017

dimento clín a perco pectivas dos pacientes nas decisões de saúde relacionadas a obesidade e terapias de controle de peso.

Devido à crescente prevalência da obesidade, são necessárias mais pesquisas sobre o custo-eficácia dos serviços de gerenciamento de pacientes obesos graves na atenção primária e secundária e para avaliar este serviço em outras localidades.

Jennings et al. ${ }^{20} \quad$ Pubmed

Clinical Obesity Oserviço de gerenciamento de peso de Fakenhamé um modelo de como um nível 3 de serviço pode ser entregue em um ambiente de cuidados primários e alcançar resultados comparáveis aos das intervenções publicadas de populações semelhantes.

As intervenções de atenção primária dadas aos pacientes com o objetivo de reduzir peso são subutilizadas, e o acompanhamento para determinar seu sucesso é ruim. Isso pode indicar uma falta de acesso do paciente para se apropriar de

Booth et al ${ }^{21} \quad$ Pubmed

BMJ open

intervenções de controle de peso corporal. Informações de saúde pública sobre os riscos para a saúde associados à obesidade devem ser oferecidas na atenção primária, onde os médicos têm a oportunidade de alcançar um grande número de pacientes e utilizar estratégias de tratamento preventivas e reativas.

Este estudo, que parece ser o primeiro a examinar a trajetória de QVRS (qualidade de vida relacionada à saúde) com esses grupos de doenças numa perspectiva comparativa, descobriu que os participantes com obesidade mórbida aumentaram sua QVRS em praticamente todas as áreas durante o período de acompanhamento de um ano após terem frequentado um curso de mestria. As pessoas com obesidade mórbida mostraram trajetórias mais positivas de QVRS do que pessoas com DPOC.

Os resultados indicam que SOC (senseofcoherence) pode ser uma medida de resultado útil para pessoas com obesidade mórbida e possivelmente pessoas com

Morbid obese adults increased their sense of coherence one year after a patient education course: a longitudinal study

Fagermoenet al ${ }^{23} \quad$ Journal of Journal of
Multidisciplina

Healthcare

BDENF

Revista de

Trabalho em equipe: em busca de um cuidado qualificado ao obeso
Nicolau et al. ${ }^{24}$ quisas que incluam subdimensões SOC são necessárias para pessoas com doenças que exploram essas inter-relações e sua ligação comsaúde.

Pelo fato de a obesidade ser uma doença multifatorial, é necessária uma equipe

2017 multiprofissional,especializada e com atuação interdisciplinar, para um atendimento qualificado de obesos grau III.

Fonte: Elaboração própria. 
nados ao tema da pesquisa, o que tornou possível formular as categorizações temáticas.

Dos 11 artigos analisados, 3 foram desenvolvidos na Noruega, 3 no Reino Unido, 2 nosEstados Unidos da América, 1 no Canadá, 1 na Espanha e 1 no Brasil. Apenas um artigo foi publicado em português, e todos os outros foram publicados em inglês. Em relação ao ano das publicações, houve incidência de 4 artigos em 2015, outros 4 em 2017, enquanto 2016 contou com duas publicações e 2014 com uma. Todos os artigos analisados abordaram a temáticado tratamento de obesos grau III.

\section{DISCUSSÃO}

Diante da análise dos artigos selecionados e das evidências científicas encontradas, consideramos relevante a apresentação de três temáticas: rede de apoio aos usuários em tratamento; a atenção primária à saúde e atividades em grupo no tratamento de obesos grau III; implementação de serviços especializados e qualificados no atendimento de obesos graves.

\section{Rede de apoio aos usuários em tratamento}

Os artigos encontrados ${ }^{16,17,19,20,24}$ fazem alguma referência à importância da rede de apoio a obesos grau III, tanto dos profissionais e unidades de saúde quanto dos familiares e de outros participantes dos programas de perda de peso.

O estudo de Sharman et al. ${ }^{16}$ refere que muitos homens e mulheres falaram em sentir-se amparados por pessoas importantes, como parceiros, amigos ou filhos durante o período de tratamento. No entanto, há relatos de familiares que, além de não apoiarem, tentavam sabotar e desencorajar os esforços do indivíduo que almejava a perda de peso. É de suma importância a família estar inserida no tratamento desse usuário, pois os hábitos alimentares de todos acabam sendo modificados por um dos membros da família estar implicado em um programa de perda de peso ou de pré-bariátrica. As compras de casa, priorizando alimentos para uma dieta saudável, a quantidade de alimentos à disposição no ambiente familiar, assim como a forma de preparo dos alimentos são exemplos de mudanças importantes para auxiliar o indivíduo que está com a intenção de perder peso.

Os participantesdo estudo de Homer et al. ${ }^{17}$ também relataram como suas famílias não entenderam sua luta em perder peso e viram a cirurgia como uma opção fácil ou suave. Referem também falta de apoio dos profissionais de saúde em seus casos. Essa falta de compreensão do profissional de saúde e das pessoas mais próximas a esses usuários os faz sentir cada vez mais marginalizados.

Para alcançar a perda de peso tão esperada, o usuário deve estar disposto a mudar seus hábitos alimentares, pois a cirurgia bariátrica não modifica a vontade de comer nem os tipos de alimentos escolhidos na dieta do cotidiano a que esses usuários estão acostumados. Entretanto, embora os esforços individuais sejam de suma importância e prioritários durante o tratamento de perda de peso, a rede de apoio é de grande valor para esses indivíduos e muitas vezes contribui para a decisão de seguir ou desistir.

Participantes de grupos focais do estudo de Sharman et al. ${ }^{16}$ falaram sobre o quão útil foi compartilhar experiências com outros participantes e como esse grupo pode beneficiar quem estava na fila de espera para cirurgia bariátrica por meio de apoio e encorajamento.

Sharman et al ${ }^{16}$ relataram também uma aparente falta de apoio e insatisfação dos participantes com a interação com os profissionais de saúde por perceberem que havia falta de compreensão, por às vezes receberem conselhos inadequados (por exemplo, recomendação de alimentos inacessíveis e conselhos conflitantes entre os profissionais de saúde) e em virtude de comentários inadequados sobre seu peso ou circunstâncias clínicas.

O estudo de Homer et al. ${ }^{17}$ aponta a necessidade de incentivar os usuários a assumirem a responsabilidade por seu comportamento alimentar. No caso da cirurgia bariátrica, por exemplo, comumente referida como uma ferramenta para controlar a alimentação e o peso, acabam sendo criadas altas expectativas de melhora no bem-estar físico, emocional e relacional. No entanto, alguns pacientes de cirurgia bariátrica não conseguem sustentar a perda de peso. Enquanto alguns usuários reconhecem que o controle pessoal é necessário, outros mantêm as expectativas irreais que eliminariam a decisão de comer ou não. Havia esperanças irrealistas entre os participantes do estudo de que eles poderiam manter algum comportamento atual e ainda perder peso após a cirurgia.

Esse incentivo vindo da equipe de profissionais de saúde também é valioso, e são necessários compreensão e preparo para lidar com esse público.O processo de perda de peso é algo difícil e muitas vezes sofrido para esses indivíduos, sendo importante valorizar cada esforço e a perda de peso, mesmo que pequena. Nessa questão, o profissional de saúde tem um papel fundamental de incentivador e apoiador.

Para Wee et al. ${ }^{19}$, é preciso destacar os benefícios da modesta perda de peso para esses indivíduos, dado que a manutenção dessa perda é difícil de alcançar e requer um compromisso. É importante que os pacientes acreditem que o peso que estão trabalhando é difícil de alcançar, e mantê-lo é realmente algo valioso. É necessário também trabalhar com esses usuários que mesmo uma modesta perda de peso (de $5 \%$ a $10 \%$ ) produz melhorias tanto nas comorbidades como na 
hipertensão arterial e valores de HbA1c (hemoglobina A1c), assim como em fatores de risco cardiovascular ${ }^{19,20}$.

Nesse contexto, o trabalho em equipe se torna essencial para auxiliar a minimizar os danos à saúde desses pacientes e iniciar um processo de mudança de estilo de vida, que necessita ser duradouro, visto que a obesidade é uma doença crônica ${ }^{24}$.

Sharman et al. ${ }^{16}$ concluem que muitos desses obesos graves podem se beneficiar do apoio psicológico e dietético e do apoio para melhorar ou pelo menos manter os níveis de atividade física e a mobilidade geral ao participarem de cursos ou tratamentos com equipe multiprofissional e de atividades grupais.

\section{A atenção primária à saúde e atividades em grupo no} tratamento de obesos grau III

Poucos autores ${ }^{14,18,20,22,23}$ versam diretamente sobre atividades educativas em grupo, o que reafirma a justificativa do estudo de que sãonecessárias mais pesquisas sobre essa temática, pois poucas publicações abordam a importância de profissionais atuarem no tratamento a obesos grau III por meio de atividades de educação em saúde em grupo como uma ferramenta para auxiliar nesse cuidado.

Entretanto, a necessidade de tratamento especializado para esse público, com abordagem multidisciplinar, e a importância do apoio da rede familiar e dos profissionais são aspectos que aparecem no discurso de vários autores entre os selecionados $^{14-18,20,22,23,24}$.

A atenção primária é, sem dúvida, o local ideal para fornecer o tratamento que visa à perda de peso, e, tendo em vista a crescente prevalência da obesidade,são necessárias mais pesquisas sobre o custo-efetividade dos serviços de controle de peso para pacientes complexos em ambos os cuidados, primários e secundários ${ }^{20}$.

Por ser a porta de entrada dos usuários, a APS é de suma importância por poder identificar esses indivíduos obesos, iniciar o tratamento e fazer os encaminhamentos necessários, isto sem perder o vínculo e mantendo o cuidado paralelo a outros serviços e necessidades desses indivíduos.

Booth et al. ${ }^{21}$ defendem que a consistência das intervenções de saúde pública sobre os riscos associados à obesidade deve ser promovida na atenção primária, onde se tem a oportunidade de chegar a um grande número de pacientes e utilizar o tratamento preventivo, bem como estratégias reativas.

Espallardo et al. ${ }^{15}$, Jennings et al. ${ }^{20} \mathrm{e}$ Booth et al. ${ }^{21}$ chamam a atenção para o fato de que a utilização de recursos de saúde aumenta com a gravidade do excesso de peso e obesidade quando comparada com outros grupos de IMC (maior pro- babilidade de ser hospitalizado ou exigir outros serviços de saúde, como laboratórios e exames de imagens) e ressaltam a importância das intervenções da atenção primária no objetivo de reduzir o peso e os agravos de saúde desses indivíduos além do enfrentamento da obesidade.

É também na APS que se encontra maior facilidade de implementar grupos de educação esaúde voltados a esse público com vistas à interação social e ao apoio, como discutido anteriormente.

Sharman et al. ${ }^{16}$ perceberam no estudo que os participantes de grupos de educação em saúde voltados para esse público falaram positivamente sobre o apoio que receberam dos outros participantes e o quão útil foi compartilhar experiências durante o período em que permaneceram no grupo de apoio ou aguardavam a cirurgia.

Grohe Urbancic ${ }^{18}$ afirmam que a interação social e o apoio oferecido durante a intervenção comportamental para mudança do estilo de vida com o objetivo de perda de peso podem ser responsáveis por melhorias iniciais na qualidade de vida relacionada à saúde.

Nesse sentido, as atividades de educação em saúde em grupo surgem como uma possibilidade de intervenção para atuar nesse contexto a fim de somar e colaborar com o tratamento desse perfil de usuários.

Implementação de serviços especializados e qualificados no atendimento de obesos graves

No Brasil, com o aumento de pessoas com obesidade grave, percebe-se que o número de vagas para cirurgia bariátrica é insuficiente e que inúmeras pessoas aguardam o procedimento, que em muitos casos pode nem acontecer. Apesar do aumento significativo do número de cirurgias realizadas a cada ano e do número de centros credenciados, ainda é incerto o período de espera em fila para realização da cirurgia bariátrica ${ }^{26}$.

Por isso, é necessária a atuação de uma equipe multidisciplinar especializada e capacitada, com abordagem interdisciplinar da obesidade, devido à complexidade das alterações encontradas, sendo utilizadas no tratamento intervenções grupais e/ou individuais, podendo ser necessário inclusive o uso de medicação.

Nicolau et al. ${ }^{24}$ afirmam ser necessário pensar em estratégias para atender esses usuários que, apesar de terem indicação para se submeter a intervenção cirúrgica, muitas vezes não o conseguirão e irão permanecer vagando pelo sistemade saúde ${ }^{24}$.

Não existem ainda relatos de modalidades de tratamentos da obesidade que promovam o mesmo nível de alcance terapêutico da intervenção cirúrgica ${ }^{25}$. Sharman et al. ${ }^{16}$ afirmam 
que intervenções não cirúrgicas para perda de peso provavelmente não são eficazes para a maioria dos usuários com obesidade grau III. Para isto, é importante uma abordagem integral realizada por médico cirurgião, endocrinologista, enfermeiro, nutricionista, psicólogo, fisioterapeuta, entre outros, de forma a compreender os diferentes aspectos que envolvem esse tipo de indivíduo ${ }^{24}$.

No estudo de Lerdalet al. ${ }^{14}$, os autores mostraram que a saúde física dos participantes melhorou consideravelmente durante o período de dois anos de acompanhamento em um curso sob medida, cujo objetivo era ajudar os participantes a tomar decisões mais fundamentadas sobre a necessidade de cirurgia ou tratamento alternativo para alcançar um estilo de vida mais saudável e melhorar sua qualidade de vida relacionada à saúde. Esse curso incluía informações sobre os tratamentos disponíveis, suas possíveis complicações e consequências para a saúde física e mental e a importância das mudanças no estilo de vida.

Já Bonsaksenet al. ${ }^{22}$ e Fagermoenet al. ${ }^{23}$ afirmam que indivíduos obesos demonstraram melhora clinicamente significante na atividade física e em todos os subdomínios da qualidade de vida relacionada à saúde após participarem de um programa de educação sem cirurgia bariátrica por 12 meses.

No entanto, não há padrões de quanto tempo de intervenção no estilo de vida seria necessário para produzir grandes efeitos. Com base em pesquisas atuais nessa área, a fase de intervenção de um programa de perda de peso no estilo de vida variou de dez semanas a 24 meses $^{18}$.

Homer et al. ${ }^{17}$ referem em seu estudo que, segundo os participantes, os profissionais de saúde que os atenderam de início avaliaram seu peso culpando-os pela obesidade e condições de saúde relacionadas; também comentaram que foram recebidos sem julgamento pela equipe especializada em obesidade e que o tratamento deveria ser assim como para vícios, e não limitado no tempo.

O cuidado a obesos grau III tornou-se um desafio para os profissionais de saúde em diversos âmbitos. Muitas vezes, o usuário ouve que a resolução do seu problema seria emagrecer, que o problema não é da especialidade procurada, e, assim, ele fica vagando pelos serviços de saúde sem acolhimento e atendimento qualificado. Este tipo de atitude não deveria ser adotado na prática de nenhum profissional ${ }^{24}$.

A obesidade é uma doença estigmatizada, que gera sentimento de impotência e vergonha nos indivíduos. Por isso, é necessário encaminhar esses obesos graves para tratamento em serviços especializados em obesidade, e eles precisam contar com o apoio da família e dos profissionais que os acompanham durante o tratamento.
Assim, vê-se a necessidade não só de uma equipe multiprofissional, mas também que esses profissionais sejam qualificados com uma prática e discurso integrados com enfoque no obeso grau III.

\section{CONCLUSÃO}

Com base nos artigos selecionados, conclui-se que a função da equipe de saúde em relação ao cuidado desse tipo de usuário vai além de orientações e informações. É necessário conscientizar o usuário a assumir o papel de protagonista do seu tratamento. As atividades educativas em saúde em grupo são ferramentas importantes para a promoção de autonomia e o compartilhamento de experiências na busca por qualidade de vida relacionada à saúde. Além disso, são imprescindíveis profissionais qualificados na composição de uma equipe multidisciplinar que atue de forma interdisciplinar no atendimento a esse público, visto que existem inúmeros entraves, que vão da estrutura física das unidades de saúde ao cuidado direto do indivíduo obeso.

O fato de os estudos efetuados confirmarem que a atividade em grupo é um instrumento possível e relevante no tratamento de obesos grau III e a importância dos profissionais serem capacitados para lidarem no cuidado desses indivíduos, torna ainda mais relevante que se volte o olhar para esse segmento da obesidade. A existência de poucos estudos realizados no Brasil justifica o investimento nesta temática, quer no contexto teórico, quer no contexto da prática.

\section{REFERÊNCIAS}

1. World Health Organization. Obesity and overweight.Geneva: WHO; 2016a. Disponível em: https://www.who. $\mathrm{int} /$ news-room/fact-sheets/detail/obesity-and-overweight. Acesso em: 18 de Dez. 2018

2. Brasil. Ministério da Saúde. Secretaria de Vigilância em Saúde. Departamento de Vigilância de Doenças e Agravos não Transmissíveis e Promoção da Saúde. Vigitel Brasil 2018: vigilância de fatores de risco e proteção para doenças crônicas por inquérito telefônico: estimativas sobre frequência e distribuição sociodemográfica de fatores de risco e proteção para doenças crônicas nas capitais dos 26 estados brasileiros e no Distrito Federal em 2018 / Ministério da Saúde, Secretaria de Vigilância em Saúde, Departamento de Vigilância de Doenças e Agravos não Transmissíveis e Promoção da Saúde. - Brasília: Ministério da Saúde, 2018.https:/ / portalarquivos2.saude.gov.br/images/pdf/2019/julho/25/vigitel-brasil-2018.pdf .Acesso em: 21 de out. 2019.

3. Nicolau IR. Atuação do enfermeiro em equipe multiprofissional no cuidado a obesos grau III / Ian Rigon Nicolau. 
- Dissertação (Mestrado Profissional em Enfermagem Assistencial) - Universidade Federal Fluminense, 2015.

4. Brasil. Ministério da Saúde; Secretaria de Atenção à Saúde, Departamento e Atenção Básica. Política Nacional de Atenção Básica [Internet]. Brasília: MS; 2012. Disponível em: http://189.28.128.100/dab/docs/publicacoes/geral/ pnab.pdf. Acesso em: 12 de Jun. 2018.

5. Van Dillen SM, Noordman J, van Dulmen S, Hiddink GJ. Quality of weight-loss counseling by Dutch practice nurses in primary care: an observational study. Eur J Clin Nutr. 2015;69(1):73-8. DOI: 10.1038/ejcn.2014.129

6. Association of perioperative registered nurses (AORN). Bariatric Surgery Guideline. AOURN Journal, v. 79, n. 5, p. 1026-52, 2004. Disponível em: http://www.ncbi.nlm.nih. gov/pubmed/15176451. Acesso em: 12 de Nov 2018.

7. World Health Organization. Report of a WHO consultation on obesity. In: Obesity: preventing and managing the global epidemic. p. 265, 2000. Disponível em: Acesso em: 9 de Jun. 2018.

8. Zimerman DE. Fundamentos básicos das grupoterapias. Porto Alegre: Artes Médica. 2. ed. 2007

9. Silva MA, de OliveiraAGB, Mandú ENT, Marcon SR. Enfermeiro \& grupos em PSF: possibilidade para participação social. RevistaCogitareEnfermagem, Curitiba, v. 11, n. 2, p. 143-149, maio/ago. 2006.

10. Brasil. Ministério da Saúde. HumanizaSUS: equipes de referência e apoio matricial. Brasília: Ministério da Saúde; 2004. Disponível em: http://picica.dominiotemporario. com/apoio\%20matricial\%20cartilha.pdf. Acesso em: 18 jul 2018.

11. Brasil. Ministério da Saúde. Secretaria de Vigilância em Saúde. Secretaria de Atenção à Saúde. Política Nacional de Promoção da Saúde. Ministério da Saúde, Secretaria de Vigilância em Saúde, Secretaria de Atenção à Saúde. 3. ed. Brasília: Ministério da Saúde, 2010. Disponível em: http:/ / bvsms.saude.gov.br/bvs/publicacoes/politica_nacional_ promocao_saude_3ed.pdf Acesso em: 11 de Nov de 2018.

12. Souza MT, Silva MD, Carvalho R. Revisão integrativa: o que é e como fazer. Einstein. 2010, v. 8, n.1, p. 102-126. Disponivel em: http://pt.scribd.com/doc/56528038/A2-Revisao-integrativa-o-que-e-e-como-fazer\#scribd. Acesso em 19 de Set de 2018.

13. Joanna Briggs Institute. Joanna Briggs Institute reviewers manual: 2011 edition. Adelaide, Australia: Author.

14. Lerdal A, Gay CL, Bonsaksen T, Fagermoen MS. Health Qual Life Outcomes. 2017; 15: 103. Predictorsofphysicaland mental health in personswithmorbidobesityattending a patienteducationcourse - a two-year follow-up study. Disponível em : https://hqlo.biomedcentral.com/articles/10.1186/ s12955-017-0675-z.Acesso em: 13 de Nov 2018.

15. Espallardo O, Busutil R, Torres A, Zozaya N, Villoro R, Hidalgo-Vega Á. OBES SURG (2017) 27: 2058. The Impact of Severe Obesity on Healthcare Resource Utilisation in Spain. Disponível em: https://doi.org/10.1007/s11695017-2596-7. Acesso em: 13 de Nov 2018.

16. Sharman MJ, Venn AJ, Jose KA, Williams D, Hensher M, Palmer AJ, et al. (2016). The supportneedsofpatientswaiting for publiclyfundedbariatricsurgery - implications for healthserviceplanners. ClinicalObesity, 7(1), 46-53. doi:10.1111/cob.12169. Disponível em: https://onlinelibrary.wiley.com/doi/abs/10.1111/cob.12169. Acesso em: 13 de Nov 2018.

17. Homer CV, Tod AM, Thompson AR, Allmark P, Goyder E.Expectationsandpatients' experiencesofobesity prior tobariatricsurgery: a qualitativestudy. BMJ Open 2016;6:e009389. doi:10.1136/bmjopen-2015- 009389. Disponível em: https://bmjopen.bmj.com/content/bmjopen/6/2/e009389.full.pdf . Acesso em: 13 de Nov de 2018.

18. Groh CJ, Urbancic JC. (2015). The Impact of a Lifestyle Change Program on the Mental Health of Obese Under-Served African American Women. Archives of PsychiatricNursing, 29(2), 76-82. Disponível em: doi:10.1016/j. apnu.2014.11.001. http://isiarticles.com/bundles/Article/pre/pdf/30907.pdf, Acesso em: 19 de Nov 2018.

19. Wee CC, Hamel MB, Huskey KW, Davis RB. (2016). The value of health and weight loss among primary care patients with moderate to severe obesity: Do quality of life factors have a larger influence than comorbidities?. Obesityresearch\&clinicalpractice, 11(1), 123-126. Disponível em https:/ / www.ncbi.nlm.nih.gov/pmc/articles/PMC5592719.Acesso em: 22 de Nov 2018.

20. Jennings A, Hughes CA., Kumaravel B, Bachmann MO, Steel N, Capehorn M, et al. (2014). Evaluation of a multidisciplinary Tier 3 weight management service for adults with morbid obesity, or obesity and comorbidities, based in primary care. Clinicalobesity, 4(5), 254-66. Disponível em https://www.ncbi.nlm.nih.gov/pmc/articles/ PMC5592719/Acesso em: 22 de Nov 2018.

21. Booth HP, Prevost AT, Gulliford MC. Access to weight reduction interventions for overweight and obese patients in UK primary care: population-based cohort study. BMJ Open 2015;5:e006642. doi: 10.1136/bmjopen-2014-006642. Disponível em https://bmjopen.bmj.com/content/5/1/ e006642.citation-tools.Acesso em: 22 de Nov 2018.

22. Bonsaksen T, Fagermoen MS, Lerdal A. Trajectories of physical and mental health among persons with morbid 
obesity and persons with COPD: a longitudinal comparative study. Journal of Multidisciplinary Healthcare, 191. doi:10.2147/jmdh.s102630. (2016). https: https://www. ncbi.nlm.nih.gov/pubmed/27175082. Acesso em 25 Nov 2018.

23. Fagermoen MS, Hamilton G, Lerdal A. Morbid obese adults increased their sense of coherence 1 year after a patient education course: a longitudinal study. Journal of Multidisciplinary Healthcare, 157. doi:10.2147/jmdh. s77763. 2015.2018. https://www.dovepress.com/morbid-obese-adults-increased-their-sense-of-coherence-1-year-after-a--peer-reviewed-article-JMDH. Acesso em 25 de Nov 2018.

24. Nicolau IR, Espirito Santo FH, David FM. Trabalho em equipe: em busca de um cuidado qualificado ao obeso / Teamwork: in searchofqualifiedcare for theobese. Rev. enferm. UFPE on line;11(1):152-159, jan.2017 . file://C:/ Users / garritano/Desktop / artigos\%20para\%20dicussão\%20completos/artigos\%20incluidos\%20e\%20excluídos/artigo\%2024.pdf. Acesso em 25 Nov 2018.

25. Sjostrom L. Review ofthekeyresultsfrom theSwedishObeseSubjects (SOS) trial - a prospectivecontrolledinterventionstudyofbariatricsurgery. J InternMed [Internet]. 2013 [cited 2014 June 20];273(3):219-34 Availablefrom: http:/ / onlinelibrary.wiley.com/doi/10.1111/j oim.12012/pdf

26. Carmo I, Fagundes MJ, Camolas J. Cirurgia Bariátrica. Rev PortCir [Internet]. 2008 [cited 2014 May 19];2(4):4350. Availablefrom: http://revista.spcir.com/index.php/ spcir/artic le/view/264/263

\section{CONTRIBUIÇÃO DOS AUTORES}

Raquel de Lima Soeiro: Mestranda no Mestrado Profissional em Ensino em Saúde, responsável pelo projeto, participou de todo planejamento, da construção científica, execução e confecção do manuscrito.

Prof ${ }^{\mathrm{a}}$. Dr ${ }^{\mathrm{a}}$ Geilsa Soraia Cavalcanti Valente: Orientadora e supervisora do projeto, participou da construção científica do manuscrito, da redação e das etapas de leitura e revisão.

Prof ${ }^{\mathrm{a}}$. Dr ${ }^{\mathrm{a}}$ Elaine Antunes Cortez: Participou das etapas de leitura e revisão.

Livia Mendes Mesquita: Mestranda no Mestrado Profissional em Ensino em Saúde participou das etapas de leitura e revisão.

Simone Costa da Matta Xavier: Mestranda no Mestrado Profissional em Ensino em Saúde participou das etapas de leitura e revisão.

Bianca Maria Innocencio da Silveira Lobo: Mestranda no Mestrado Profissional em Ensino em Saúde participou das etapas de leitura e revisão.

\section{CONFLITO DE INTERESSES}

Os autores declaram não possuir nenhum conflito de interesse, atual e potencial, de ordem financeira, pessoal e/ou institucional

\section{ENDEREÇO PARA CORRESPONDÊNCIA}

Rua Barata Ribeiro, 147 / 402 - Copacabana, Rio de Janeiro-RJ CEP: 22011-001 